\title{
Diagnostic Ultrasound Safety
}

\author{
${ }^{1}$ Kazuo Maeda, ${ }^{2}$ Asim Kurjak
}

\begin{abstract}
Ultrasound bioeffect is discussed from its physical property, i.e. thermal effect by thermal index, mechanical effect by mechanical index, and by the output intensity of ultrasound. Generally, thermal and mechanical indices should be lower than 1 in obstetrical setting, and threshold output intensity of no bioeffect is lower than SPTA $240 \mathrm{~mW} / \mathrm{cm}^{2}$ in pulse wave. Pulsed Doppler ultrasound thermal and mechanical indices should be also lower than 1 , and should be carefully used it in 11 to $13^{+6}$ weeks of pregnancy. Real-time B-mode, transvaginal scan, pulsed Doppler, 3D and 4D ultrasound were separately discussed in the ultrasound safety. Generally diagnostic ultrasound is safe for the fetus and embryo, if thermal and mechanical indices are lower than 1, and ultrasound devices are safe, if it is used under official limitation, e.g. the output intensity is less than SPTA 10 $\mathrm{mW} / \mathrm{cm}^{2}$ in Japan. The ultrasound user is responsible ultrasound safety, e.g. higher thermal and mechanical indices than 1 should be lowered to be lower than 1 , controlling the device output intensity. The user should learn bioeffects of ultrasound and prudent use of ultrasound under the ALARA principle.
\end{abstract}

Keywords: Ultrasound diagnosis, Ultrasound user, Ultrasound safety, Pulsed Doppler, B-mode, Three-dimensional, fourdimensional, Transvaginal scan, Thermal index, Mechanical index, Neuron migration, Ductus venosus, First trimester.

How to cite this article: Maeda K, Kurjak A. Diagnostic Ultrasound Safety. Donald School J Ultrasound Obstet Gynecol 2014;8(2):178-183.

\section{Source of support: Nil}

Conflict of interest: None

\section{INTRODUCTION}

Although no adverse effect on human subject has been reported in diagnostic ultrasound exposure, rumors were circulated worrying over the possible damage or teratogenicity of diagnostic ultrasound comparing to the X-ray diagnosis. However, ultrasound is a sound which is the mechanical vibration of propagating media. Its frequency is too high to resonate by human ear, so that it is inaudible by human. The ultrasound, of which frequency is several

\footnotetext{
${ }^{1,2}$ Professor

${ }^{1}$ Department of Obstetrics and Gynecology, Tottori University Medical School, Yonago, Japan

${ }^{2}$ Rector of DIU, Libertas International University; Department of Obstetrics and Gynecology, Zagreb University, Zagreb, Croatia

Corresponding Author: Kazuo Maeda, Professor, Department of Obstetrics and Gynecology, 3-125, Nadamachi, Yonago Tottoriken 683-0835, Japan, Phone and Fax: 81-859226856 e-mail:maedak@mocha.ocn.ne.jp, j2p4e4@bma.bigobe.ne.jp
}

$10 \mathrm{kHz}$, can resonate with the ear of some animals, by which they communicate each other, or measure the distance to a hindrance with the purpose to avert it, where ultrasound is natural range finder in the case, i.e. ultrasound is useful tool to keep the wild life healthy, but not be hazardous. However, ultrasound intensity is extraordinary larger than human voice in the diagnostic devices, that mean medical ultrasound of diagnostic device should be used by appropriate intensity. If too large is the intensity, there may exist some bioeffect in young tissues of fetus and neonate. 'Not too much' is the principle to use diagnostic devices. The ALARA (as low as reasonably achievable) principle will show this idea in the prudent use of ultrasound, i.e. ultrasound intensity is as low as possible, if the ultrasound diagnosis is achieved, e.g. the ultrasound intensity is as low as $1 \mathrm{~mW} / \mathrm{cm}^{2}$ in TOITU actocardiograph, which records fetal heart rate and movement in fetal monitoring. Ultrasound user is requested to prudently handle the ultrasound devices, because the user is responsible to the ultrasound safety. ${ }^{1,2}$

\section{GENERAL PRINCIPLE}

\section{Thermal Effect and Thermal Index (TI)}

The ultrasound beam is absorbed by tissues in the propagation to reflect from the tissue to form ultrasound image, but at the same time the tissue is slightly heated and elevates the temperature of the tissue by the absorbed ultrasound. The elevated temperature is a tool to measure ultrasound effect. Meanwhile in the biology, actively heated animal fetus developed head and neck anomalies in experiments. The anomaly highly developed when the temperature was high and the exposure time was long (Fig. 1). ${ }^{3}$ In ultrasound, the tissue heating is high when the ultrasound intensity is high and exposure time is long. Therefore, the ultrasound bioeffect is indirectly expressed by the temperature elevation and exposure time. However, as the ultrasound is attenuated during propagation, and the heat is cooled by local perfusion, standard attenuation and perfusion are added in the temperature measurement of exposed subject. By this way, the ultrasound intensity to rise local temperature for $1^{\circ} \mathrm{C}$ was defined as 1 thermal index. Thus, thermal effect of diagnostic ultrasound was defined in TI 1 to TI 6 (Fig. 2). Temperature elevation was 1 to $6^{\circ} \mathrm{C}$, and actual temperature was 38 to $43^{\circ} \mathrm{C}$. Safe exposure time is 1,000 minutes ( 17 hours) if TI is 1 and $1 \mathrm{~min}$ if TI is 6 according to Figures 1 and 2. Since fetal bone absorbs 


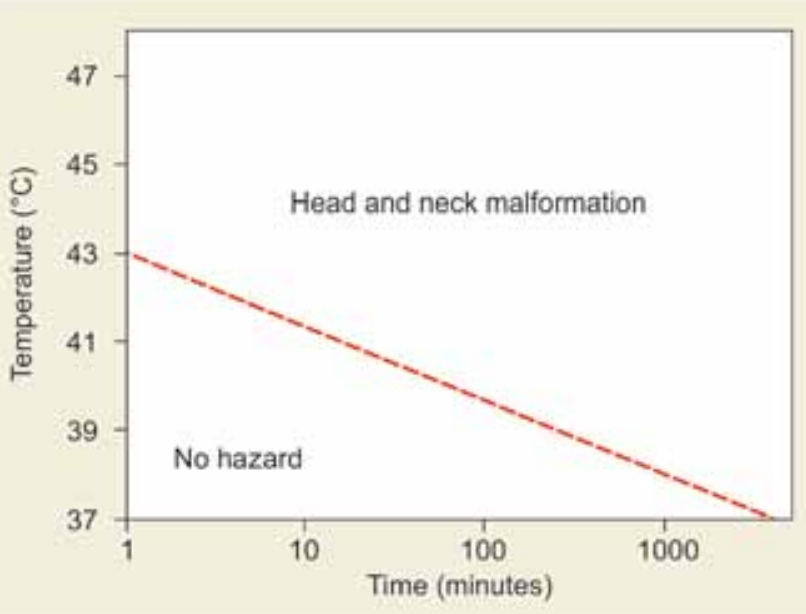

Fig. 1: The effect of active fetal animal heating. Head and neck malformation developed by long exposure to high temperature ${ }^{3}$

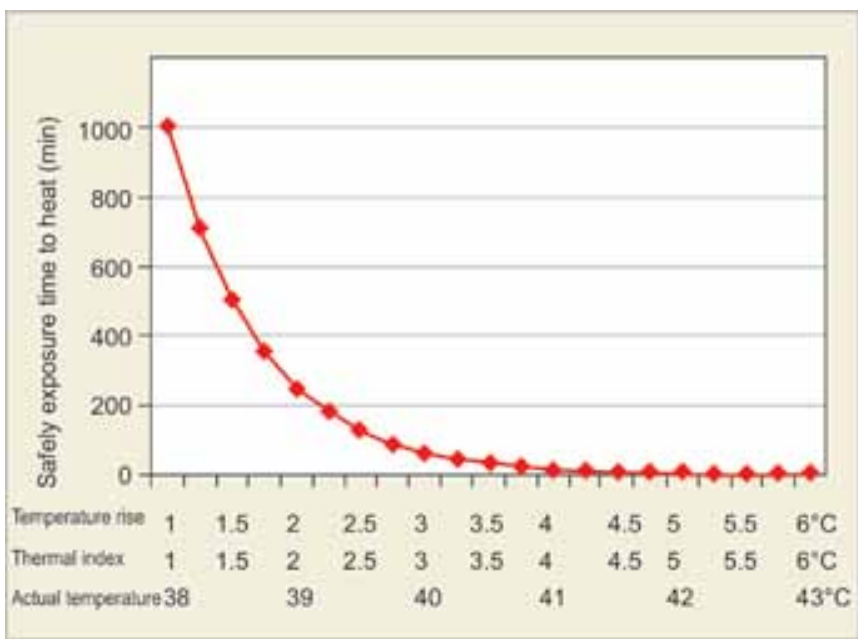

Fig. 2: The relations among the temperature elevation higher than $37^{\circ} \mathrm{C}$, thermal index and active temperature

more ultrasound and temperature elevation was higher than soft tissue, TI is divided into TIs, TIb and TIc. TIs is soft tissue TI, TIb is bone TI, and TIc is cranial TI. TIs is used in the embryonal stage before fetal bone formation, and TIb is used after 10 or more weeks of pregnancy after fetal bone formation. TIc is used in the transcranial scan.

Caution should be paid for the temperature of the tissue exposed to diagnostic ultrasound in febrile patients, where the body temperature is already higher than $37^{\circ} \mathrm{C}$. For example, if TI is 2 in $38^{\circ} \mathrm{C}$ febrile patient, the temperature rise above physiologic condition is $3^{\circ} \mathrm{C}$, the situation is the same as TI 3 in nonfebrile normal temperature case, and therefore, permitted ultrasound exposure time is too short to achieve correct diagnosis.

\section{Mechanical Effect and Mechanical Index}

The mechanical index (MI) is used for the estimation of mechanical bioeffect of ultrasound. The MI is rarefactional sound pressure (Pr) expressed in Megapascal (MPa)
(Figs $3 \mathrm{~A}$ and B) divided by square root of ultrasound frequency determined in $\mathrm{MHz}$. The large negative pressure pulse forms vacuum bubbles, of which collapse (cavitation) accompany high pressure, high temperature and forms free radical. Although the bubble is not formed in the cell plasma due to its high viscosity, and the free radical formed in the liquid hardly reaches cells due to its short life, the high pressure of pulse waves may produce any bioeffect, therefore, even simple imaging devices should be carefully handled in the relation to mechanical effect. Therefore, it will be important to use the device, of which MI is less than 1. Since the hemorrhage is found in neonatal animal lung after the exposure to intense ultrasound, lower MI than 1 is recommended in neonatal examination at the chest.

Biological effects of acoustic streaming, capillary blood cell stasis by the standing wave, or the direct ultrasonic pressure requires further basic studies.

It is requested to ultrasound user to lower the output intensity of diagnostic ultrasound devices and keep the TI and MI lower than 1, when the monitor displays TI or MI higher than 1 , because the user is responsible to the ultrasound safety.

\section{REAL-TIME B-MODE}

The real-time B-mode is the most principal tool among the clinical ultrasound devices. Its use at maternal abdominal surface (abdominal scan) is the most common technique in obstetrics. Its TI and MI are 1 or less in obstetrical setting. The world federation of ultrasound in medicine and biology (WFUMB) ultrasound safety and bioeffect symposium stated that simple B-mode device without additional function is not concerned on the thermal effect of ultrasound, because of its very low output intensity. Although temporally averaged ultrasound intensity of B-mode ultrasound imaging is as low as continuous wave $(\mathrm{CW})$ ultrasound below $10 \mathrm{~mW} / \mathrm{cm}^{2}$ in Japanese Industrial Standard (JIS), ${ }^{4}$ the peak intensity of PW used in the B-mode is as high as several $10 \mathrm{~W} / \mathrm{cm}^{2}$ (see Figs $3 \mathrm{~A}$ and $\mathrm{B}$ ). Thus, mechanical bioeffect is high in PW, e.g. the lowest intensity to suppress the growth curve of cultured cells were SPTA (special peak, temporal average) $240 \mathrm{~mW} /$ $\mathrm{cm}^{2}$ in PW and $1,000 \mathrm{~mW} / \mathrm{cm}^{2}$ in $\mathrm{CW}$, and there was no cell growth suppression below the intensity levels. ${ }^{5}$ The results were authorized by Japan society of ultrasonics in medicine (JSUM), and it was the base of JIS regulation. Also we accepted the thermal index, because the TI 1 output power was $210 \mathrm{~mW} / \mathrm{cm}^{2}$ by our calculation, which was almost the same to the bioeffect threshold of PW in our study.

Although there was no limitation of exposure time to commercial real-time B-mode ultrasound, recent study reported the disturbance of neuronal migration of fetal 


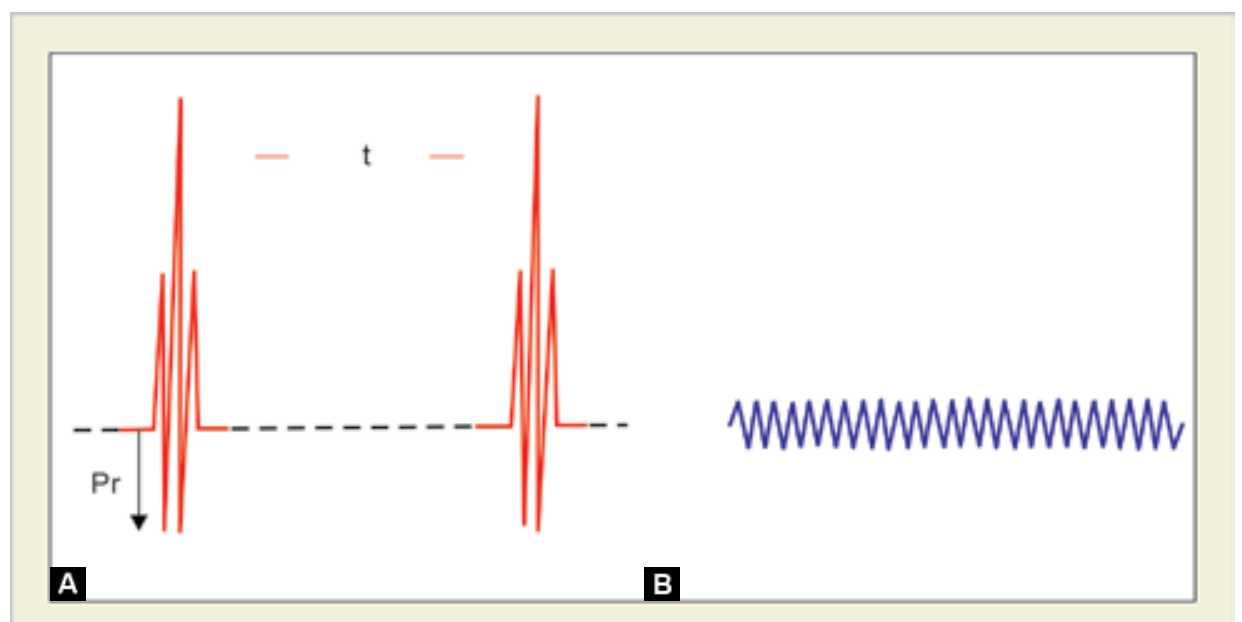

Figs $3 A$ and $B$ : Two kinds of ultrasound waves in diagnostic ultrasound: $(A)$ pulse wave $(P W)$ and $(B)$ continuous wave (CW). Pr: Peak rarefactional pressure; PRF: Pulse repetition frequency $=1 / \mathrm{t}(\mathrm{sec})$

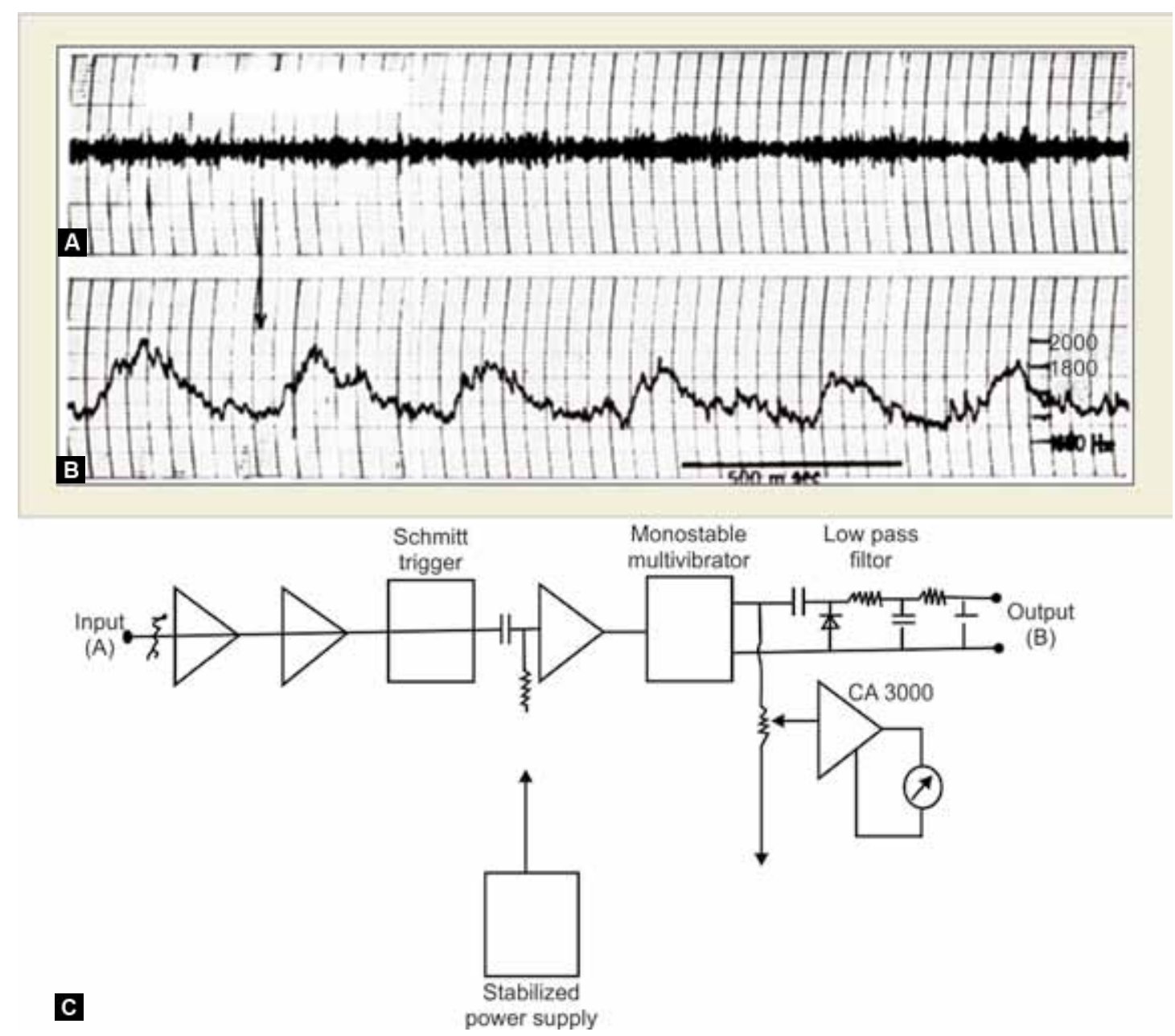

Figs 4A to C: The first CW Doppler fetal arterial flow wave reported in 1969': (A) Continuous wave Doppler fetal heart detector (Doptone) output Doppler sound of fetal arterial flow, (B) fetal arterial Doppler flow wave obtained by the frequency demodulation, using the $\mathrm{C}$ circuit, (C) frequency demodulation circuit hand-made by $\mathrm{K} \mathrm{Maeda}^{9}$

mice exposed to commercial B-mode after exposure of fetal mice for more than 30 minutes. ${ }^{6}$ Ultrasound organizations recommended not to prolong exposure to fetal brain. However, the presence of any opportunity to elevate fetal temperature was wondered in this report, because the transducer heat was not insulated by constant temperature water, but the transducer was directly attached pregnant animal via ultrasound conducting material. Since 
transvaginal transducer surface temperature is limited to be below $41^{\circ} \mathrm{C}$, abdominal scan transducer can be heated some degree over $37^{\circ} \mathrm{C}$, and I remember animal fetal head anomaly was produced by the direct attachment of transducer to pregnant animal abdomen, but there was no anomaly by the exposure to ultrasound through the temperature stabilized water. According to the NCRP report 20 minutes heating of fetal animal the anomaly developed when the fetus was heated to $41^{\circ} \mathrm{C}$ or higher temperature (see Fig. 1). ${ }^{3}$ It may be possible to heat fetal animal to some degree to produce a functional change of fetal brain by 30 minutes exposure to the heated transducer without heat insurance in the report of abnormal neuronal migration of fetal animal by 30 or more minutes attachment of active B-mode transducer. ${ }^{6}$

\section{TRANSVAGINAL SCAN}

\section{Transducer Temperature}

The transducer head of transvaginal scan is smaller than abdominal scan transducer, while the transvaginal image should be as clear as abdominal scan. Therefore, the triggering electric current of small transvaginal probe will be similar to large abdominal scan probe, so that the temperature elevation will be higher in transvaginal than abdominal scan. It may be a reason why transvaginal scan transducer surface temperature is requested to be lower than $41^{\circ} \mathrm{C}$, while no limitation of transducer temperature is requested in abdominal scan, but the abdominal transducer temperature may be also higher than physiologic body temperature. Transvaginal scan user should be careful on the direct heating of the attached tissue due to high surface temperature of the transducer, which directly attach vaginal wall, and the subjects are closer to the transducer in transvaginal scan than the abdominal. The transducer temperature displayed on the monitor screen should be lower than $41^{\circ} \mathrm{C},{ }^{1}$ because of possible hazard to attached sensitive tissue by the heated transducer. The user of every intracavitary scan should be also careful on the transducer temperature.

\section{Thermal and Mechanical Indices}

The ultrasound bioeffect should be particularly careful when the study subject is embryo or fetus. The heat purely induced by the ultrasound is shown in the thermal index displayed on diagnostic ultrasound monitor screen. The output intensity of transvaginal scan should be reduced by the user, if the TI or MI is larger than 1, which should be lower than 1 by the lowering control of diagnostic device. Although MI is shown by the negative pressure of ultrasound pulse also in the transvaginal scan, we should also aware the positive pulse pressure of which absolute value may be the same or more than $\operatorname{Pr}$ (negative pressure), particularly in pulsed Doppler, even in transvaginal scan. Although MI is determined by the negative pressure of ultrasound pulse, positive pressure is parallel to negative one, and therefore, positive pressure will be also represented by the MI.

\section{DOPPLER ULTRASOUND}

The important ultrasound bioeffect is thermal effect due to temperature rise induced by ultrasound absorption, because malformations were reported after the exposure of animal embryos and fetuses to high temperature in biological experiments. ${ }^{3}$ No hazardous thermal effect is expected when the temperature rise of exposed tissue is less than $1.5^{\circ} \mathrm{C}$, and local temperature is lower than $38.5^{\circ} \mathrm{C} .{ }^{1}$ Five minutes' duration of $41^{\circ} \mathrm{C}$ temperature can be hazardous to the tissue. Inertial cavitation and other mechanical effects are concerned in the nonthermal bioeffects of ultrasound.

\section{Intensity of Doppler Ultrasound}

No hazardous thermal effect is expected in common B-mode imaging device because of its minimum heat production due to low ultrasound intensity, therefore, WFUMB concluded that the use of simple imaging equipment is not contraindicated on thermal grounds. ${ }^{1}$ The real-time B-mode, simple three-dimensional (3D) and four-dimensional (4D) imaging devices, ultrasonic fetal heart beat detector and fetal monitor are included in the category. Diagnostic ultrasound safety was perfect in Japan, after the regulation of Japanese Industrial Standard (JIS) on the output power of diagnostic ultrasound devices below $10 \mathrm{~mW} / \mathrm{cm}^{2}$ in $1980,{ }^{4}$ where the level was $1 / 24$ of hazardous $240 \mathrm{~mW} / \mathrm{cm}^{2}$ of pulse wave (PW) ultrasound. ${ }^{5}$ However, ultrasound safety has been discussed again after the introduction of Doppler flow velocity measurement, because pulsed Doppler (spectral Doppler) method utilized higher power than B-mode ultrasound. There are two kinds of Doppler, i.e. pulsed wave Doppler (pulsed Doppler) and continuous wave Doppler (CW Doppler) (see Figs 3A and B). Ultrasound intensity is low in CW Doppler (Figs 4A to C), but it did not indicate the location of flow measurement, whereas the measurement location is indicated by the sample volume in pulsed Doppler, thus the pulsed Doppler is the use in obstetrics. Concern on pulsed Doppler ultrasound is limited in fetal Doppler study, and the Doppler study is not concerned in maternal blood flow, e.g. uterine arterial flow, or in umbilical cord blood flow. It is concerned if the fetal body is exposed to the pulsed Doppler ultrasound beam during the studies on blood flow in maternal or umbilical cord vessels. Since the pulsed Doppler flow velocity wave is a sequential vertical 
displays of frequency spectrum of flow pulsed Doppler signal, it needs a high ultrasound intensity, if compared to B-mode imaging. Maximum intensity of commercial Doppler ultrasound in adult reaches $3 \mathrm{~W} / \mathrm{cm}^{2}$, which is definitely higher than the intensity of B-mode, where it is the level of ultrasound physiotherapy for the tissue heating. The difference between the therapeutic ultrasound and adult pulsed Doppler is the exposure time, i.e. it is short in Doppler flow measurement and long in therapeutic ultrasound. Ultrasound safety is concerned in the physiotherapy, of which transducer should be always moved on the bone, and young bone and pregnant woman are contraindicated to ultrasound physiotherapy. Therefore, thermal effect is big concern in pulsed Doppler ultrasound bioeffect. Temperature rises not only at the sample volume, but also in all tissues passed by the Doppler ultrasound beam.

Recently, transient increase of apoptotic index of fetal liver tissue was reported 7 hours after the exposure of fetal ductus venosus for 20 or more seconds to pulsed Doppler ultrasound, of which TI and MI were lower than 1, in fetal rat. ${ }^{7}$ Bioeffects and Safety Committee of international society of ultrasound in obstetrics and gynecology (ISUOG) ${ }^{8}$ declared their statement on the safe use of Doppler in the 11 to $13^{+6}$ weeks fetal ultrasound examination as follows:

1. Pulsed Doppler (spectral, power and color flow imaging) ultrasound should not be used routinely.

2. Pulsed Doppler ultrasound may be used for clinical indications such as refine risks for trisomies.

3. When performing Doppler ultrasound, the displayed thermal index (TI) should be $\leq 1.0$ and exposure time should be kept as short as possible (usually no longer than 5-19 minutes) and should not exceed 60 minutes.

4. When using Doppler ultrasound for research, teaching and training purposes, the displayed TI should be $\leq 1.0$ and exposure time should be kept as short as possible (usually no longer than 5-10 minutes) and should not exceed 60 minutes. Informed consent should be obtained.

5. In educational settings, discussion of first trimester pulsed or color Doppler should be accompanied by information on safety and bioeffects (e.g. TI, exposure times and how to reduce output power).

6. When scanning maternal uterine arteries in the first trimester, there are unlikely to be any fetal safety implications as long as the embryo/fetus lies outside the Doppler ultrasound beam.

\section{THREE-DIMENSIONAL ULTRASOUND}

The gray level data is acquired in $3 \mathrm{D}$ imaging by repeated moving scans of real-time B-mode array transducer, the scans are completed until whole imaging volume in a few seconds, the image data are stored in the computer memory, and the unique $3 \mathrm{D}$ images are processed in the computer after fetal or embryonic ultrasound exposure. A point of fetal body would be exposed to ultrasound not frequently in whole scans. Therefore, 3D ultrasound exposure at a point of the fetus or embryo and the bioeffects caused by ultrasound would be the same as a simple B-mode. Also scanning time is as short as seconds. Accordingly, possible temperature rise and thermal effect in $3 \mathrm{D}$ ultrasound are almost the same as simple B-mode, thus 3D technique will be safe in the thermal effect that was not concerned in the B-mode ultrasound exposure.

Since fetal 3D image is obtained by the B-mode pulse wave ultrasound, its instantaneous mechanical effect is equal to simple B-mode. Mechanical bioeffect is concerned, if power Doppler is combined 3D ultrasound, while the mechanical effect is the same as B-mode imaging, if no pulsed Doppler is combined, i.e. its mechanical effect is determined by its instantaneous temporal peak (TP) intensity or sound pressure, represented by mechanical index (MI), which is recommended to be lower than 1 in fetal or embryonal studies, because pulmonary hemorrhage is induced in animal neonates by exposure to intense pulsed ultrasound, possibly by the mechanical effect to the air contained pulmonary tissue.

The safe character of 3D ultrasound, however, will be changed to the situation to concern the bioeffects, when the pulsed Doppler is combined to 3D, i.e. the safety is ruled by the intensity of pulsed Doppler, where an ultrasound user should follow the description in the chapter 'Doppler Ultrasound'.

\section{FOUR-DIMENSIONAL ULTRASOUND}

Although the 4D ultrasound image is obtained by computer processing of 10 to 24 frames of fetal 3D pictures in a second, most fetal parts are not expected to be exposed to ultrasound repeatedly, because the fetus is moving and therefore a fixed fetal part changes its position by fetal motion. Thermal effect of ultrasound will not be concerned in 4D, despite large number of ultrasound scan is irradiated, because simple B-mode is the base of $4 \mathrm{D}$ images, and thermal effect is not concerned in the B-mode. The 4D ultrasound is considered to be long B-mode scan which is divided into short pieces of scans in second and minute, where the heating by ultrasound absorption, if any, is cooled by the circulation. In addition, even if there as repeated exposure of a fixed fetal part, e.g. in fetal resting state, ultrasound bioeffect is not accumulated in intermittent exposure.

Therefore, there will be no problem caused by ultrasonic thermal effect in $4 \mathrm{D}$ surface rendering. There will be also no limitation of ultrasound examination, if the thermal index 
(TI) is less than 1 . As for the safety of mechanical effect of pulsed ultrasound, 4D ultrasound examination may not be hazardous to the fetus or embryo, when the MI is less than 1. Since recent study of B-mode exposure to fetal mice, fetal brain neuron migration was disturbed by the exposure for such long time as 30 minutes, ${ }^{6}$ it is recommended also in 4D ultrasound not to study fetal brain for very long duration.

The safety of 4D ultrasound is ruled by the combination of pulsed ultrasound too, and the description in the chapter of 'Pulsed Ultrasound' should be followed.

\section{NONMEDICAL FETAL IMAGING}

An ultrasound image should be obtained just medical indication, but some parents get fetal 3D or 4D images in pregnancy for keepsake and souvenir of their children by nonmedical indication. Since diagnostic ultrasound safety is achieved by the study to know ultrasound bioeffects, prudent use of ultrasound devices and the control of ultrasound output intensity to keep the TI and MI at safe level by trained ultrasound user after detailed training course. It may be unsafe to handle ultrasound imaging devices by the persons who received no training on possible ultrasound bioeffects, prudent use of diagnostic devices and the control of device output intensity. Therefore, nonmedical use of diagnostic ultrasound imaging devices by untrained staff would be prohibited to the healthy development of the babies.

\section{CONCLUSION}

Diagnostic ultrasound safety is achieved by controling the output pulse and continuous waives using thermal index and mechanical index, which will be kept at 1 or less in abdominal scan, transvaginal scan, pulsed Doppler ultrasound, 3D and 4D ultrasound. The lowest SPTA intensity of pulse wave ultrasound to suppress cultured cell growth was $240 \mathrm{~mW} / \mathrm{cm}^{2}$, below which there was no effect of ultrasound. An ultrasound user is trained to know possible ultrasound bioeffects, thermal and mechanical indices, how to lower the indices higher than one displayed on the monitor screen, prudent use of ultrasound under ALARA principle, because the user is responsible to ultrasound safety.

\section{REFERENCES}

1. WFUMB symposium on safety and standardization in medical ultrasound. Issues and recommendations regarding thermal mechanisms for biological effects of ultrasound. Hornbaek, Denmark, 30 August-1 September 1991. Ultrasound Med Biol 1992;18:731-810.

2. American Institute of Ultrasound in Medicine/National Electrical Manufacturers Association; Standard for Real Time Display of Thermal and Mechanical Acoustic Output Indices on Diagnostic Ultrasound Equipment. 1992.

3. National Council on Radiation Protection and Measurements (NCRP). Exposure Criteria for Medical Diagnostic Ultrasound: I. Criteria based on Thermal Mechanisms. NCRP Report No. 113,1992

4. Maeda K, Ide M. The limitation of the ultrasound intensity for diagnostic devices in the Japanese Industrial standards. IEEE Trans Ultrasonics, Ferroelectrics and Frequency Control, 1986; UFFC-33:241-244.

5. Maeda K, Murao F, Yoshiga T, Yamauchi C, Tsuzaki T. Experimental studies on the suppression of cultured cell growth curves after irradiation with $\mathrm{CW}$ and pulsed ultrasound. IEEE Trans Ultrasonics, Ferroelectrics and Frequency Control, 1986; UFFC-33:186-193.

6. Ang ESBC Jr, Glucic V, Duque A, et al. Prenatal exposure to ultrasound waves impacts neuronal migration in mice. www. pnas.org/cgi/doi/10.1073/pnas06052934103.

7. Pellicer B, Herraiz S, Taboas E, et al. Ultrasound bioeffects in rats: quantification of cellular damage in the fetal liver after pulsed Doppler imaging. Ultrasound in Obstet Gynecol 2011; 37:643-648.

8. Bioeffects and Safety Committee, ISUOG statement on the safe use of Doppler in the 11 to $13^{+6}$ weeks fetal ultrasound examination. Ultrasound Obstet Gynecol 2011;37:625-628.

9. Maeda K, Kimura S, Nakano H, et al. Pathophysiology of fetus. Fukuokab Printing, Fukuoka. The 21st Annual Meeting of Japanese Obstetrical and Gynecological Society, Kanazawa, April 14, 1969. 